\title{
Clonorchiasis: An emerging foodborne disease of public health concern
}

\section{Mahendra Pal}

Narayan Consultancy on Veterinary Public Health and Microbiology,Aangan,Jagnath Ganesh Dairy Road,Anand-388001,Gujarat,India

Corresponding author: Mahendra Pal, Narayan Consultancy on Veterinary Public Health and Microbiology, Aangan, Jagnath Ganesh Dairy Road, India, E-mail: palmahendra2@gmail.com

Received date: January 2, 2020; Accepted date: January 10, 2020; Published date: January 13, 2020.

Citation: Mahendra Pal. (2020) Mahendra Pal. Clonorchiasis: An emerging foodborne disease of public health Concern. J. Nutrition and Food Processing, 3(1); DOI: 10.31579/2637-8914/021

Copyright: (C) 2020. Mahendra Pal. This is an open-access article distributed under the terms of the Creative Commons Attribution License, which permits unrestricted use, distribution, and reproduction in any medium, provided the original author and source are credited.

\begin{abstract}
Foodborne diseases caused by multiple etiologic agents are prevalent worldwide affecting both sexes and all age groups. Clonorchiasis is considered as an emerging major foodborne disease of public health problem as about 35 million people are infected globally. It is a foodborne helminthic zoonosis of a greater socioeconomic importance in several countries of Asia. The disease is caused by Clonorchis sinensis, which is a trematode. The infection occurs by the consumption of raw or inadequately cooked fish contaminated with metacercaria of the parasite. The clinical spectrum of disease in humans depends on the burden of parasites. In severe infections, jaundice, cholangitis, ascites, cholecystitis, formation of gall stone, and gastrointestinal bleeding are observed. Maximum cases are observed in males than females, and infection is serious in elderly persons. The diagnosis of disease can be confirmed by parasitological technique. Presently, praziquantel is the drug of choice as it has shown high cure rates in endemic areas. The education campaigns regarding the consumption of properly cooked fish and avoidance of faecal matter to ponds as a fish food are practical strategies that may help to control the disease.
\end{abstract}

Keywords: clonorchiasis; emerging foodborne; fish; public health,trematode

\section{Introduction}

Foodborne diseases have emerged as significant growing public health and economic problem. These diseases can occur in sporadic and epidemic form causing morbidity as well as mortality in susceptible population, and are observed in developing and developed countries of the world [1]. Currently, more than 250 diseases of multiple etiologies are described from many regions of the world [2].There are many emerging foodborne parasitic diseases, such as cryptosporidiosis, giardiosis, toxoplasmosis, cyclosporasis, trichinellosis, fascioliasis, echinococcosis, taeniasis, cysticercosis, fasciolopsiasis, clonorchiasis, gnathostomiasis, sparganosis, paragonimiasis, diphylobothriasis, anisakiosis etc., that are transmitted through contaminated food and water [3,1] Very recently, Pal and co-workers (2018) presented a systematic review on the public health importance of foodborne helminthisis. In this context [4] has mentioned that international community has neglected foodborne trematode infections as their impact has not been well studied. Clonorchiasis, also known as Chinese liver fluke disease, Oriental liver fluke, is an important foodborne helminthic zoonosis of public health importance and is caused by Clonorchis sinensis that parasitizes the human intra-hepatic bile duct $[5,6]$ The history of clonorchiasis goes back to year 1874 when a pathologist first described a new species of liver fluke in the bile ducts of a Chinese during autopsy [7].The disease is recorded from many Asian countries, such as China, Hong Kong, Japan, Korea, Philippines, Taiwan, and Vietnam [5,8,9,10] Maximum cases of clonorchiasis are reported from China [11]. It is estimated that about 600 million people are at a risk of acquiring $C$. sinensis infection [12]. It is important to mention that out of 35 million cases occurring globally, 15 million originated from China
[11]. In this context, Lai and co-investigators (2016) presented the current status of clonorchiasis in China. It is recognized as a helminthic zoonosis of major socio-economic importance in East Asia, including China, Korea, and Vietnam.

The climate change has significant implications on the emergence and reemergence of many diseases of diverse etiologies [13].The impact of climate on clonorchiasis is studied by [14] in Guangzhou, China. These investigators reported that an increase in temperature and rainfall can augment the risk of infection due to $C$. sinensis whereas a rise in relative humidity may decrease the threat of $C$. sinensis infection. Moreover, climatic factors have an effect on the occurrence and transmission of clonorchiasis that may be helpful for developing an early warning system. The resistance of microbes to various drugs has become a global public health concern. The increase in the prevalence of antimicrobial resistance makes the hunt for new antibiotic agents crucial. Antimicrobial peptides (AMPs) from natural resources have been acknowledged as appropriate tools to fight antibiotic-resistant bacteria. The study conducted by [15] reported that Clonorchis sinensis living in germ-filled environments could be a good source of antimicrobials. These investigators proposed a refined computational method to develop new AMPs and identify four AMP candidates, which could serve as templates for further development of peptide antibiotics. Further research on this subject may be rewarding.

The transmission of infection occurs when humans ingest raw infected fresh fish containing metacercaria, the infective form of parasite, [5] The infection rate is usually higher in males than in females, and is most serious in elderly people [16] Most cases of clonorchiasis occur in lowor middle-income countries of Asia $(\mathrm{Pal}, 2014)$. Disease has also been observed in travelers returning from endemic regions [1] Persons who have the habit of eating raw or undercooked fish show higher infection 
rates compared to those who do not consume raw fish. In addition to human beings, natural infection has also been recorded in animals, such as cats, dogs, pig, camel, mink, badger, and weasel [5]. The present paper delineates the emerging significance of clonorchiasis as an important foodborne helminthiasis of public health concern.

\section{Clnical Spectrum}

The patient may show no clinical symptoms or exhibit general malaise, abdominal discomfort, diarrhea, fever, chills, anorexia, hepatic cirrhosis, icterus, hepatomegaly, oedema, ascites, gastrointestinal bleeding, formation of gall stones, cholangitis and cholecystitis [11,5] Complications, such as hepatitis, liver abscess, pancreatitis, and pyogenic cholangitis can occur when bile ducts are obstructed by adult parasites $[17,18]$ The chronic infection with $C$. sinensis has been associated to the development of cholangiocarcinoma and the delay in treatment may be fatal [4]. Presently, C. sinensis is recognized as a biocarcinogen, which is strongly linked to cholangiocarcinoma in human beings [19] it is pertinent to mention that the parasite can live over 25 years in humans [11].

\section{Diagnosis}

Clinical signs and history of ingestion of raw fish may help in tentative diagnosis. However, microscopic examination of stool or bile for the demonstration of ova of C.sinensis confirms the diagnosis of disease [5]. Immunological test like ELISA can support the diagnosis of clonorchiasis, but hardly differentiate between acute and past infection (Marcos et al., 2008). Multiple imaging modalities, such as ultrasonography, conventional cholangiography, and endoscopic retrograde cholangiopancreatography may help to confirm clinical diagnosis [18] it is advised that patients who present with biliary disease and having the history of consumption of raw fish should be thoroughly investigated for C.sinensis infection.

\section{Treatment}

Praziquental is considered the drug of choice for the chemotherapy of clonorchiasis [18]. Treatment with praziquental at the dosage rate of 25 mg per $\mathrm{kg}$ of body weight for 1-2 days has shown good clinical response [5]. In endemic nations, praziquental has been used as single-dose regimen for mass treatment. The drug is well tolerated, with mild adverse effects, such as dizziness, headache, and abdominal discomfort [15, 20] in addition, another antihelminthic drug named albendazole, has also successfully treated cases of clonorchiasis $[11,15]$. Tang and co-workers (2016) reported that antiparastic drug Tribendimidine with less side effects than praziquental, has demonstrated encouraging result in the management of disease. Moreover, the new strategies of combining nonpolluted fish culture with the use of fish vaccines might represent a viable alternative to block the transmission of this parasite and ensure the food safety of freshwater fish. These authors are confident that sustainable and inventive control strategies may eradicate this helminthic disease in the near future. As plant based drugs are considered very safe, it is therefore, recommended to undertake detailed study to evaluate the efficacy of herbal drugs for the treatment of clonorchiasis.

\section{Control}

Currently, no vaccine is available to control the disease. However, certain measures such as thorough cooking of fish before consumtion, avoidance of human and animal faecal matter to ponds as a fish food, treatment of night soil with ammonium sulphate to kill eggs of parasite, and eradication of snail can minimize the incidence and prevalence of disease. In addition, the health education of the fish eating community about the risk involved in eating raw fish and environmental sanitation is of paramount importance [5].

\section{Conclusion}

Clonorchiasis, an emerging important foodborne disease, is caused by C.sinensis. The disease presents a significant threat to human health, and is endemic in Asian countries including China. Climate change has an effect on the occurrence of disease. Further work on the impact of disease on human health should be assessed. It is emphasized that new technologies for better surveillance and control of foodborne parasites including C.sinensis appear necessary. As diagnosis of clonorchiasis is challenging, there is a need to develop a simple, rapid, sensitive, and low cost diagnostic test, which can be routinely performed in public health and parasitology laboratories.

\section{Acknowledgements}

The author is very grateful to Prof.Dr.R.K.Narayan for going through the manuscript and Anubha for technical help. This paper is dedicated to all the scientists who did pioneer work in foodborne parasitic diseases

\section{References}

1. Pal, M. 2014. Impact of emerging foodborne pathogens on public health. Ph.D. Lecture Notes. Addis Ababa University, College of Veterinary Medicine, Debre Zeit, Ethiopia.Pp.1-21.

2. CDC. 2009. Food safety. Centre for Disease Control. Atlanta, Georgia, USA.

3. Slifko, T.R., Smith, H.V. and Rose, J.B. 2000. Emerging parasite zoonoses associated with water and food. International Journal of Parasitology 30: 1379-1393.

4. WHO.2010. Working to overcome the global impact of neglected tropical diseases. First WHO report on neglected tropical diseases? World Health Organization, Geneva, Switzerland.

5. Pal, M. 2007. Zoonoses. Second Edition. Satyam Publishers,Jaipur,India.

6. Xu, Y., Chen, W., Bian, M., Wang, X., Sun, J., Sun, H., Jia, F., Liang, C., Li, X. and Zhou, X, Huang Y, Yu X. 2013. Molecular characterization and immune modulation properties of Clonorchis sinensis-derived RNASET2. Parasite Vectors 6: 360-10.1186/1756-3305-6-360.

7. Strauss, W.G.1962. Clonorchiasis in San Francisco. Journal of American Medical Association 179: 290- 292.

8. Dorny,P., Praet,N., Deckers,N. and Gabriel, S. 2009.Emerging foodborne parasites. Veterinary Parasitology 163: 196-206.

9. Qian, M.B., Chen, Y.D., Liang, S., Yang, G.J. and Zhou, X.N. 2012. The global epidemiology of clonorchiasis and its relation with cholangiocarcinoma. Infectious Diseases of Poverty 1: 4.

10. Gowda, C. 2015. Recognizing clonorchiasis : A foodborne illness leading to significant hepatobiliary disease. Clinical Liver Disease: A multimedia Review Journal 6: 44-46.

11. Lun, Z.R., Gasser, R.B., Lai, D.H., Li, A.X., Zhu,X.Q., Yu, X.B. and Fang,Y.Y. 2005. Clonorchiasis: a key foodborne zoonois in China. Lancet Infectious Disease 5: 31-41. 
12. Kesier, J. and Utzinger,J. 2005. Emerging foodborne trematodiasis. Emerging Infectious Diseases 11: 1507-1514.

13. Pal, M., Ayele, Y., Hadush, A, Kundu, P. and Jadhav, V. J. 2018. Public health significance of helminthiais: A systematic review. Journal of Experimental Food Chemistry 4: 1- 6.

14. Dave, S., Dave, P. and Pal, M. 2015. The impact of climate change on emergence and re-emergence of human vector diseases. International Journal of Livestock Health 5: 1- 10.

15. Li, T., Yang, Z. and Wang, M. 2014. Correlation between clonorchiasis incidences and climatic factors in Guangzhou, China. Parasite Vectors 7: 29.

16. Yoo, W.G., Lee, S., Lee, M,R., Yun, M.R., Kwon, T. And Kim, D, W. 2015. Genome-wide identification of antimicrobial peptides in the liver fluke, Clonorchis sinensis. Bioinformation. 11:17-20.

17. Qian, M.B., Chen, Y.D., Liang, S., Yang, G.J. and Zhou, X.N. 2012. The global epidemiology of clonorchiasis and its relation with cholangiocarcinoma. Infectious Diseases of Poverty 1: 4.
18. Wang, K.X., Zhang, R.B., Cui, Y.B., Tian, Y., Cai, R. and Li, C.P.2004. Clinical and epidemiological features of patients with clonorchiasis. World Journal of Gastroenterology 10: 446448.

19. Rim, H.J.2005. Clonorchiasis: an update. Journal of Helminthology 79: 269- 281.

20. Lai, D.H., Hong, X.K., Su. B.X., Liang, C., Hide, G., Zhang, X., Yu, X. And Lun, Z.R. 2016. Current status of Clonorchis sinensis and clonorchiasis in China. Transaction of Royal Society of Tropical Medicine and Hygiene 110:21-27.

21. Hong, S.T. and Fang, Y. 2012. Clonorchis sinensis and clonorchiasis, an update. Parasitology International 61: 17- 24.

22. Tang, Z., Huang, Y. and Yu, X. 2016.Current status and perspectives of Clonorchis sinensis and clonorchiasis: epidemiology, pathogenesis, omics, prevention and control. Infect Dis Poverty 5, 71 (2016)

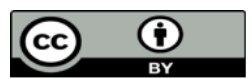

This work is licensed under Creative Commons Attribution 4.0 License

To Submit Your Article Click Here: Submit manuscript

DOI:10.31579/2641-0419/038

\footnotetext{
Ready to submit your research? Choose Auctores and benefit from:

* fast, convenient online submission

* rigorous peer review by experienced research in your field

* rapid publication on acceptance

* authors retain copyrights

* unique DOI for all articles

* immediate, unrestricted online access
}

At Auctores, research is always in progress.

Learn more www.auctoresonline.org/journals/clinical-cardiology-andcardiovascular-interventions 